\title{
Lepidopteran webber, Orthaga exvinacea oviposits amidst conspecific colonies: a social facilitation gone unnoticed
}

\author{
P. D. Kamala Jayanthi*, Vivek Kempraj and M. A. Ravindra \\ Department of Entomology and Nematology, Indian Institute of Horticulture Research, Hessaraghatta Lake Post, Bengaluru 560 089, India
}

The present study hypothesized that sequential oviposition behaviour of mango leaf webber, Orthaga exvinacea in/near conspecific webs, affects the cohort composition within the web as well as web density in a particular tree, resulting in a multicohort composition of webs and gregarious webbing in the orchard. The active $O$. exvinacea webs contained mixed cohorts of developmental stages involving eggs, larvae (I-VII instars) and pupae $\left(\mu=6.50, \sigma^{2}=55.23\right)$. Taylor's power law further supported the cohort variability with fluctuating $b$ (aggregation index) across different age groups of webs, which explained heterogeneity within the web composition. Olfactometer assays and electroantennogram studies further revealed significant differences in the response of gravid female to volatiles of different web categories. GC-EAD identified potent chemical cues, namely $(E)-2-h e x e n a l,(Z)-3-h e x e n-1-o l$, $(Z)-3$-hexen-1-ol, formate, $\beta$-ocimene, $\beta$-linalool, alloocimene, cis-3-hexenyl iso-butyrate, cis-3-hexenyl$\alpha$-methylbutyrate, $\delta$-octalactone, $\quad(+)$ - $\alpha$-copaene, methyleugenol, trans caryophyllene, (-)- $\alpha$-gurjunene, $\alpha$-humulene, (+)-aromadendrene and ledene that elicited antennal response in $O$. exvinacea female moths. Thus, the results of this study indicate that $O$. exvinacea moths oviposit in/near conspecific webs pointing to the existence of social facilitation in these moths.

Keywords: Conspecific webs, mango orchards, Orthaga exvinacea, oviposition, social facilitation.

'SOCIAL FACILITATION', a tendency to team up with other individuals of the same species (= conspecifics), is quite evident in several species of vertebrates and invertebrates. It is commonly defined as the pattern of behaviour that is initiated or increased in frequency by the presence of conspecific species ${ }^{1}$. It is a synergistic process or more precisely, a nonlinear response to sense and communicate with others. Conspecific attraction occurs when the presence of individuals of the same species increases the probability of more individuals coming to that $\operatorname{site}^{2}$.

For many years, field studies of birds have suggested that avian territories seem to be arranged in clusters, independent of resource distribution ${ }^{2}$. Similarly, social

*For correspondence. (e-mail: jaiinsect@ gmail.com) facilitation has been found in many arthropod species and occurs ubiquitously among social insects ${ }^{3-6}$. In the case of arthropods, a behaviour that may be influenced by conspecifics is oviposition site selection. The decision on oviposition site is usually taken by a female to ensure the survival and fitness of its progeny ${ }^{3}$. Oviposition in phytophagous insects is a dynamic process in which individual females respond to variation in both host quality and availability in functional ways ${ }^{3}$. In addition to this social facilitation, which is an individual's response to the presence of conspecifics, may influence the reproductive behaviour of invertebrate species. Our regular surveillance of mango leaf webber Orthaga exvinacea Hampson (Lepidoptera: Pyralidae) has yielded key insights into the cohort phenology and web composition of this important pest, hinting at the possible existence of social facilitation that was not noticed hitherto.

$O$. exvinacea, commonly found throughout the plains of southern India, usually attacks perennial trees, viz. mango (Mangifera indica) and cashew (Anacardium occidentale). The gravid female lays eggs either singly or in clusters on leaves ${ }^{7}$. On hatching, the caterpillars feed gregariously by scraping on the leaf surface and together web the tender shoots/leaves like a nest (Figure 1). As the larvae are gregarious and confined to the web, the decision of oviposition by the gravid female moths is solely responsible for the particular cohort composition within the web. In the present case, as the moth has the habit of laying eggs either singly or in clusters, it is hypothesized that in a particular web, in all probability almost similarly aged larvae must be present if only a single moth lays all the eggs.

Secondly, usually the gravid female exploits all available food resources in such a manner that its progeny have an equal chance of survival ${ }^{8}$. In the present case, the quantum of food is not a limitation as the larva feeds on leaves of the mango tree, i.e. evergreen with luxuriant foliage throughout the year. Therefore, our second hypothesis is that a female moth has ample choice to lay its eggs in a spatially partitioned manner so that the progeny can exploit abundant resources, thereby avoiding aggregation of cohorts within the web. Thirdly, the gravid moth, $O$. exvinacea may be attracted to oviposit in the coeval webs of conspecifics or near them, thus hypothesizing the practice of social facilitation in this lepidopteran webber. 


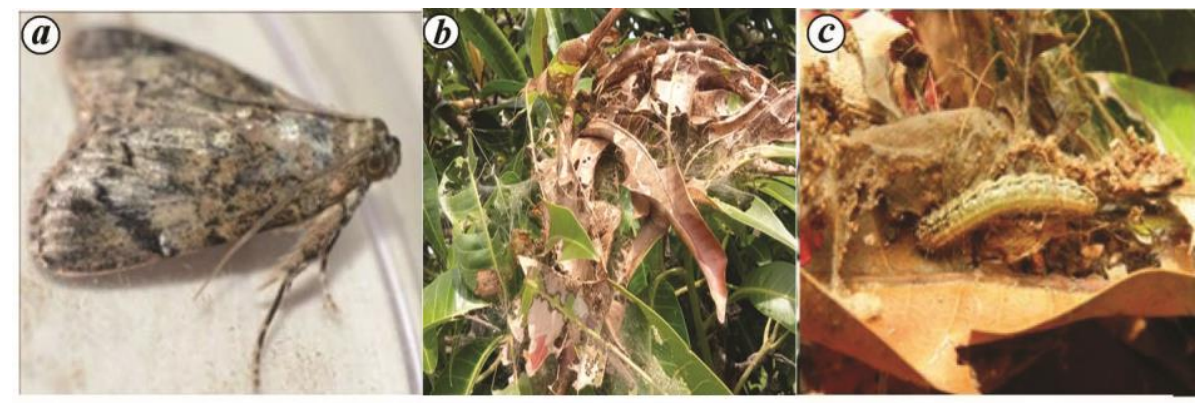

Mean cohort composition of $O$. exvinacea ( $n=97)$

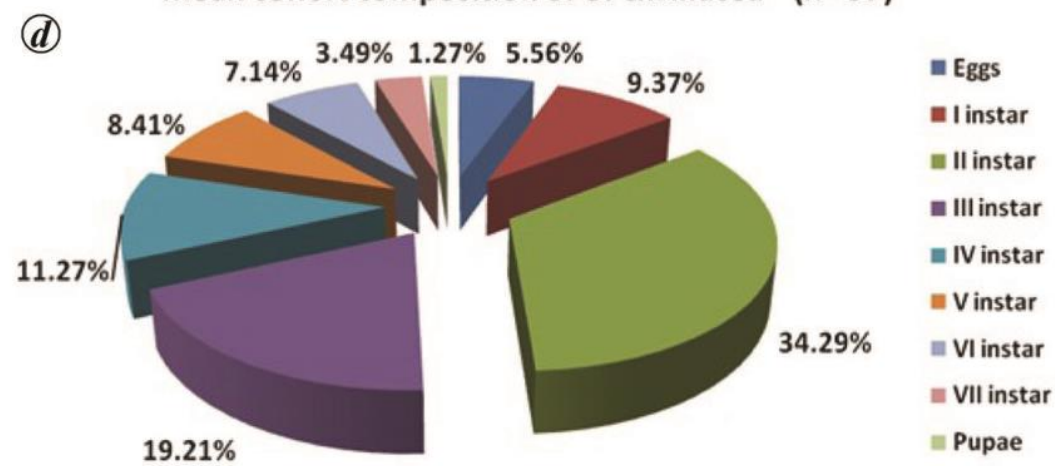

Figure 1. $\boldsymbol{a}$, Female moth Orthaga exvinacea. $\boldsymbol{b}$, Web on mango tree. $\boldsymbol{c}$, Larva within the web. $\boldsymbol{d}$, Mean cohort composition in a web $(n=97)$.

\section{Materials and methods}

\section{Study area}

The study was carried out in the mango orchards of the Indian Institute of Horticultural Research (IIHR), $\left(12^{\circ} 58^{\prime} \mathrm{N} ; 77^{\circ} 35^{\prime} \mathrm{E}\right)$, Bengaluru, during peak infestation of $O$. exvinacea.

\section{Field procedures}

Orthaga exvinacea webs were censused through multiple surveys conducted every week between August and October 2012. Locations of webs and adjacent ones in the same tree/branch were detected by visual counting.

\section{Laboratory observations}

For laboratory observations, the individual webs were collected randomly into polythene covers with least disturbance to prevent the escape of larvae. The covers were draped slowly over the webs and quickly tied with rubber bungs to avoid aggressive escape of larvae. The shoots were then cut to detach the webs from the tree using secateurs. Then, the webs were brought to the laboratory for further data collection. Before dissecting the webs, their appropriate ages were recorded based on intensity of webbing and drying of leaves by grading them on a 1-4 scale, where 1, 2, 3, 4 represent young, middle-aged, old and very old webs respectively. Later, each web was opened carefully into a plastic tray and larval composi- tion was noted. The number of different cohorts of $O$. exvinacea, namely egg, I, II, III, IV, V, VI, VII instars and pupa occurring in a particular web was recorded. The total number of larvae in each stage for each web was counted. Depending on the cohort number $\left(C^{*}\right)$ in each web, the probability of visitation by adult moths was calculated considering the life-history traits of gravid female $O$. exvinacea as a function of co-occurring $C^{*}$ and adult moth longevity.

\section{Insects}

The webs containing the larvae were brought to the laboratory and kept in plastic containers $(35 \times 15 \mathrm{~cm})$. The plastic containers were covered with clean white muslin cloth with the help of rubber bands to prevent the larvae from escaping. Fresh mango leaves were provided as and when the leaves showed withering symptoms, until pupation. These containers were periodically checked for pupae, which were collected and kept in small wooden rearing cages $(30 \times 30 \times 45 \mathrm{~cm})$ for the emergence of adults. The adults were provided with $10 \%$ honey solution using moist cotton swabs ad libitum, until ready for use in behavioural bioassays.

\section{Volatile collection}

Headspace volatiles emitted from four different categories of webs, viz. young middle-aged, old and very old were collected using an air-entrainment system according 
to the procedure described earlier ${ }^{9}$. The webs were collected with minimal disturbance from the field and brought to the laboratory. The charcoal-filtered air was pumped into a PET bag containing the webs at a flow rate of $0.81 \mathrm{~min}^{-1}$ and at the same time a portion of the air was pumped out of the bag at a flow rate of $0.51 \mathrm{~min}^{-1}$. The air pumped out was passed through a mini glass column packed with Porapak Q $(50 \mathrm{mg})$ to capture the volatile compounds. Volatiles from leaves that were absorbed onto Porapak Q at $27^{\circ} \mathrm{C}$ for $12 \mathrm{~h}$ were eluted twice with $500 \mu \mathrm{l}$ of redistilled diethyl ether.

\section{Olfactometer assays}

To study the attraction of female moths towards volatiles of different categories of webs (young, middle-aged, old, very old), behavioural bioassays were carried out using the $Y$-tube olfactometer according to the procedure described earlier ${ }^{10}$. The $Y$-tube olfactometer consisted of a $Y$-shaped glass tube $(2.5 \mathrm{~cm}$ diameter, $23 \mathrm{~cm}$ arm length and $23 \mathrm{~cm}$ common arm length) connected to two tubular glass chambers $(38 \mathrm{~cm}$ long and $6 \mathrm{~cm}$ in diameter) that served as odour sources (one on each arm). Purified air was drawn through activated charcoal into the glass chambers and $Y$-tube arms at a rate of $600 \mathrm{ml} \mathrm{min}^{-1}$ at the entrance. All parts of the olfactometer were washed in detergent solution, rinsed with acetone and finally oven-dried for at least $2 \mathrm{~h}$ at $120^{\circ} \mathrm{C}$ before using for bioassay. The experiments were conducted in an isolated darkened room $\left(26^{\circ} \pm 2{ }^{\circ} \mathrm{C}, 70 \% \mathrm{RH}\right)$ to avoid contaminant odours. The female moths of $O$. exvinacea were brought into the room $30 \mathrm{~min}$ before the start of the experiment to allow acclimatization to the room conditions. A single moth was released at the entrance of the common arm of the $Y$-tube and exposed to volatiles from different webs independently along with solvent control. The test samples $(10 \mu \mathrm{l})$ were placed on filter-paper strips and allowed to evaporate before placing them inside one of the two glass chambers connected to one of the two arms of the $Y$-tube olfactometer.

Once inside the $Y$-tube, the behaviour of each moth was observed until it made a choice for up to $10 \mathrm{~min}$. A moth was considered to have made a choice if it entered either arm and crossed a score line drawn $3 \mathrm{~cm}$ from the intersection of the tube. By contrast, a moth was considered not having made a choice if it remained in the common arm of the $Y$-tube by the end of the observation period. A new pair of filter-paper strips with fresh odour sample was used for each individual female tested. The sample size consisted of 30 adult moths.

\section{Electrophysiology studies}

Studies of the electroantennogram (EAG) activity of natural samples of all four categories of webs with female
O. exvinacea moth antennae were carried out as described earlier ${ }^{11}$. Gas chromatography electroantennodetection (GC-EAD) studies were carried out with highly preferred web volatiles against the antenna of gravid female moth according to the procedure described earlier ${ }^{9}$.

The volatiles of the highly preferred web were subjected to Gas Chromatography-Mass Spectroscopy analysis using an HP 6890 system (Agilent Technologies, USA) coupled with a mass selective detector (HP 5973; Agilent Technologies, USA) operated in electron impact mode (source temperature: $230^{\circ} \mathrm{C}$; transfer line temperature: $250^{\circ} \mathrm{C}$ ). A HP-5 MS phenyl methyl siloxane nonpolar capillary column (length: $30 \mathrm{~m}$; ID: $0.25 \mu \mathrm{m}$ ) was used. The mobile phase was helium (99.9\% pure; Praxair, India) with a flow rate of $1 \mathrm{ml}^{-1}$. Split inlet with a split ratio of $50: 1$ and temperature of $280^{\circ} \mathrm{C}$ were set before injecting the samples. Oven temperature programme was set to $70^{\circ} \mathrm{C} \mathrm{min}^{-1}$ with $2 \mathrm{~min}$ hold and a ramp of $10^{\circ} \mathrm{C}$ $\min ^{-1}$ till $260^{\circ} \mathrm{C}$. The MS detector was maintained at $280^{\circ} \mathrm{C}$. Mass spectra of GC EAD-detected compounds were compared using spectral libraries (Wiley 2012 and NIST 2012 versions).

\section{Statistical analyses}

The data were subjected to ANOVA to compare treatments on the basis of means, standard errors and CD. To examine the similarity in cohort composition of the web, we compared summary statistics for the frequency of each cohort. In addition, we performed correlation and regression analysis using the representation features, viz. age of the web and brood composition of each web to determine if the age of the web influences cohort composition.

Mean density, variance and mean crowding (tree- and web-wise) were calculated. These data were used for calculation of the power law. Taylor's linear power law regresses logarithmic variance to logarithmic mean $\left(\log \left(s^{2}\right)=\log (a)+b \log (x)\right)$, where $a$ is a sampling factor and $b$ is an index of aggregation assumed to be constant within a species. Homogeneity of regression coefficients was tested using analysis of covariance. Quantitative measurement of web diversity, evenness and richness was made using diversity indices, viz. Shannon-Weiner index $(H)$, Simpson index $\left(D_{\mathrm{s}}\right)$, cohort richness $\left(M_{\mathrm{a}}\right)$, cohort evenness $(J)$ and dispersion indices, viz. variancemean ratio $(V / M)$, coefficient of aggregation $(k)$, mean crowding index (MCI) and David-Moore index (IDM).

Results of behavioural bioassays were analysed for preference (percentage of moths that made a choice among odour sources) using $\chi^{2}$ test to test the null hypothesis of equal preference for the given odour samples. Data on EAG responses were subjected to one-way ANOVA and means were separated using critical difference with $\alpha$ set at 0.05 . 

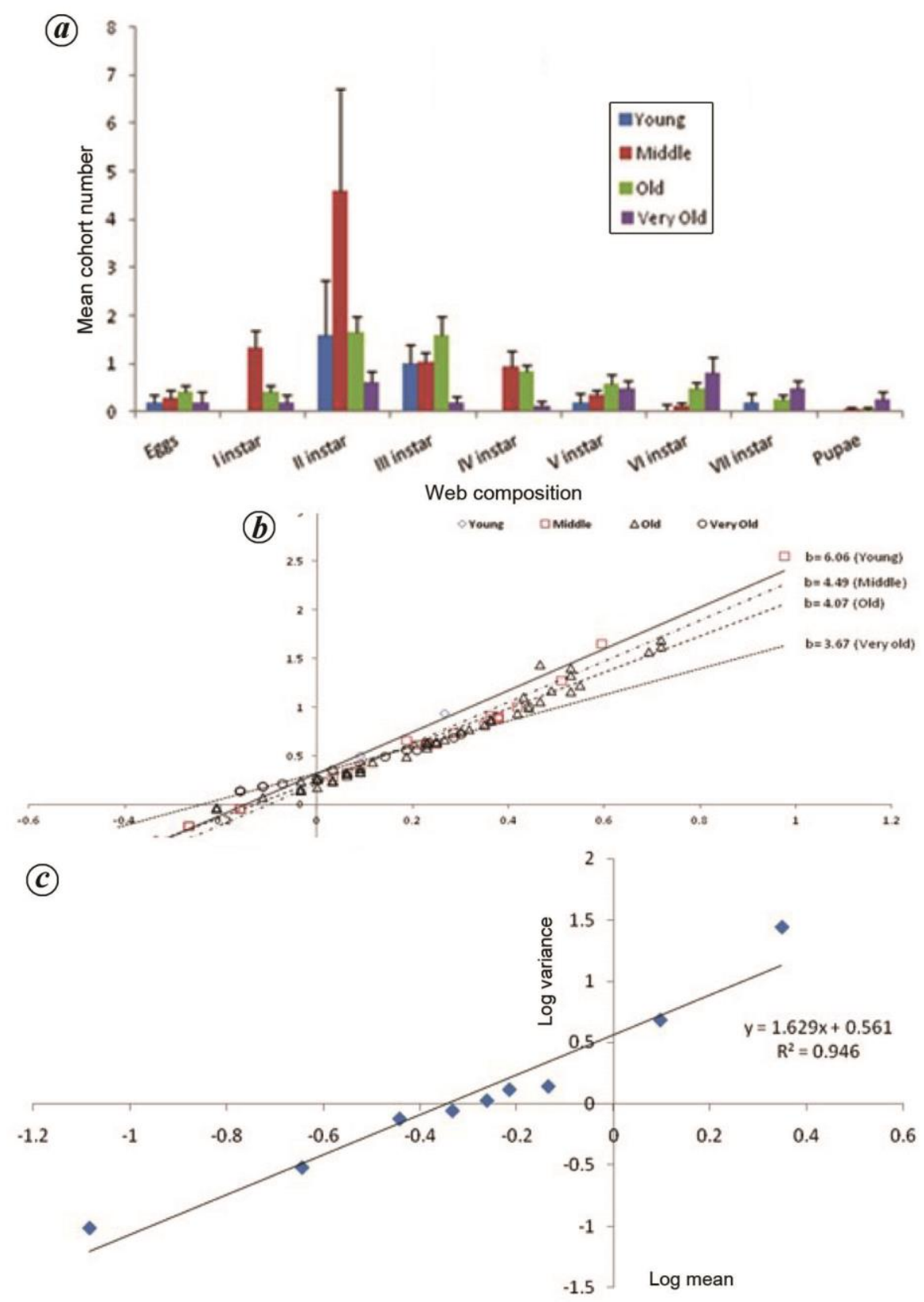

Figure 2. $\boldsymbol{a}$, Mean cohort occurrence in different age group of $O$. exvinacea webs. $\boldsymbol{b}$, Variability in the mean composition in different age groups of webs. $\boldsymbol{c}$, Variability in the mean cohort composition within a web.

\section{Results}

Analysis of frequencies, correlations, regressions and crosstabs revealed a considerable amount of multi-attraction visits by the gravid female $O$. exvinacea to the conspecific webs.

\section{Larval cohort qualitative and quantitative variability}

In each web, a mixed cohort of different phenological stages of $O$. exvinacea was found $(n=97)$. The total number of different phenological stages in a web ranged from 1.00 to 56.00 with a mean of $6.50 \pm 0.76$ and sample variance $\left(s^{2}\right)$ of 55.23 . There was significant difference between different cohorts, viz. eggs, I, II, III, IV, V, VI, VII and pupae occurring within a web $(P<0.0001$, $F=45.60$ ). Figure 2 presents the mean per cent cohort composition for different stages, viz. eggs $(0.36 \pm 0.01)$, I instar $(0.61 \pm 0.12)$, II instar $(2.23 \pm 0.53)$, III instar $(1.25 \pm 0.22)$, IV instar $(0.73 \pm 0.12), \mathrm{V}$ instar $(0.55 \pm$ $0.10)$, VI instar $(0.46 \pm 0.10)$, VII instar $(0.23 \pm 0.06)$ and pupae $(0.08 \pm 0.03)$. The mean number of cohorts (developmental stages) per web was $2.80 \pm 0.16$ and ranged

CURRENT SCIENCE, VOL. 119, NO. 5, 10 SEPTEMBER 2020 
from 1.00 to 7.00 across the webs. Similarly, there was significant difference between different age groups of webs $(\mathrm{d} f=3,192 ; F=11.27, P<0.0001)$ for cohort composition (d $f=11.192 ; F=30.77, P<0.0001)$ (Figures 1 and 2).

\section{Cohort and web composition variability}

Analysis of covariance revealed significant difference between regression slopes of $O$. exvinacea over different web groups $(F=14.33 ; P<0.001)$. This homogeneity of Taylor's power law suggested that web composition patterns of $O$. exvinacea did vary across different age groups of webs, as they were composed of multi cohorts. In Figure $2 b$, the data are heterogeneous and obey Taylor's fluctuations scaling with the characteristic exponent $b=6.06$ (young), 4.49 (middle), 4.07 (old) and 3.67 (very old) for different age groups of webs, explaining the heterogeneity of web composition change depending upon age of the web. Thus, the parameter $b$ characterizing heterogeneity of web composition changes depending on the age of the web. Young webs characterized by the largest value of $b$ (6.06), with progressive reduction in its value for the other age groups implies that web composition becomes comparatively more homogeneous with
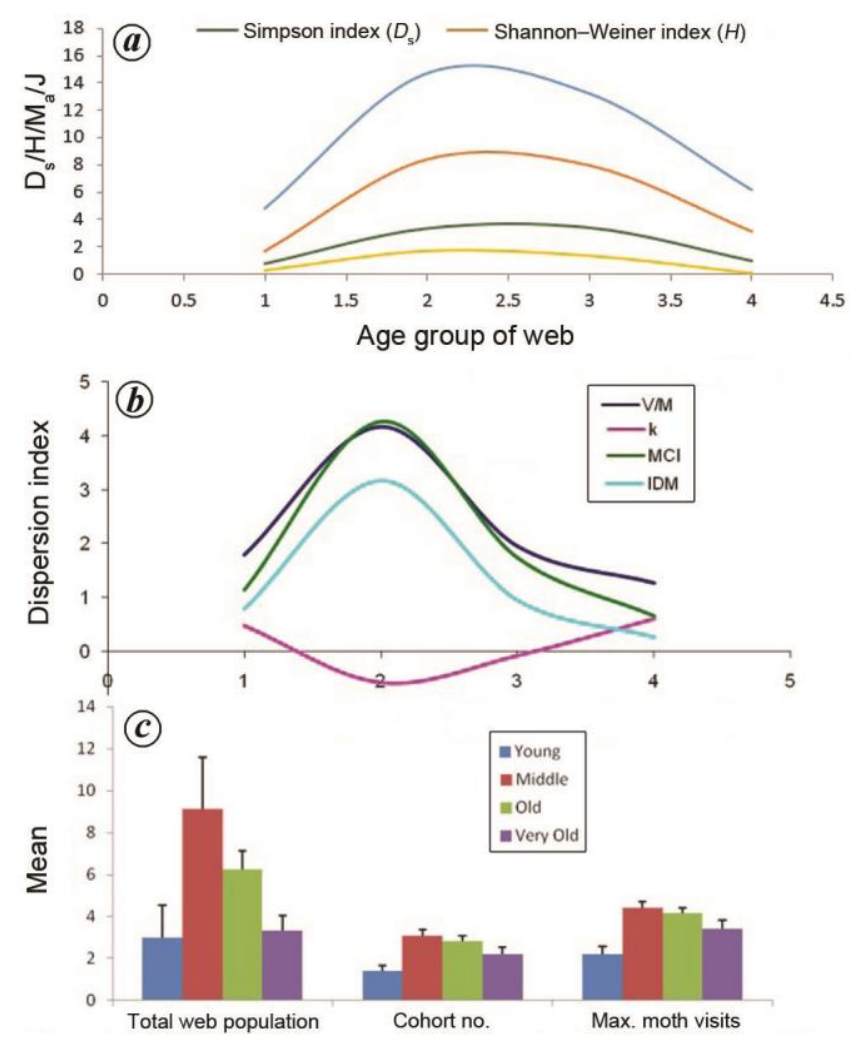

Figure 3. Comparison of $(\boldsymbol{a})$ diversity and (b) dispersion indices among different age groups of $O$. exvinacea webs. $\boldsymbol{c}$, Population differences and moth visits in different age groups of webs. time. A similar trend was noticed even within a web, suggesting multi-cohort existence $(b=1.629)$ (Figure 2).

High values of Shannon-Weiner index $(H)$ in middleaged and old webs showed more diverse and evenly distributed web composition compared to young and very old webs. Similarly, both cohort richness $\left(M_{\mathrm{a}}\right)$ and cohort evenness $(J)$ also increased with age, and later showed a downward trend to that of a typical bell-shaped curve. Simpson's diversity index $\left(D_{\mathrm{s}}\right)$, a measure of diversity which takes into account both richness and evenness also exhibited similar trend (Figure $3 a$ ). The dispersion indices, $V / M$, MCI and IDM were $>1$, suggesting a more pronounced aggregation in the middle-aged and old groups compared to young and very old webs. Similarly, a decrease in an aggregation parameter $k$ indicated an increase in aggregation, a typical negative binomial distribution (Figure 3).

\section{Occurrence of multi cohorts within a web}

The total web population and available cohorts per web showed significant differences with age of the web. The young and very old webs significantly had a smaller number of web population and cohorts compared to the middle-aged and old-aged webs. In other words, attraction of the web to gravid O. exvinacea increased with age of the web, as is evident by the moth visits, but tended to decrease later (Figure 3). A similar trend was observed with moth visits as well. The expected moth visits exhibited significant correlation with all cohorts across the webs. The regression analysis also revealed a similar trend as maximum variability $\left(R^{2}=0.75\right.$ using exponential model and 0.61 using linear model) in the population of web dwellers was explained by the expected moth visits (Figure 4; Supplementary Figure 1). The mixed cohorts across different age groups of $O$. exvinacea webs indicate sequential ovipositional behaviour of moths into the existing webs. The expected moth visits also exhibited significant positive correlation with different cohorts of $O$. exvinacea, viz. eggs $(r=0.20)$, I instar $(r=0.40)$, II instar $(r=0.20)$, III instar $(r=0.55)$, IV instar $(r=0.36)$ and $\mathrm{V}$ instar $(r=0.44)$, indicating the presence of multiple cohorts that can be attributed to multiple moth visits.

\section{Occurrence of multiple webs in a tree}

The number of webs within a tree suggested that occurrence of $>1$ web in a tree is a common phenomenon indicating the gregarious webbing nature of $O$. exvinacea. Further, the frequency of occurrence of webs followed a normal distribution indicating a continuous probability distribution. This again endorses that $O$. exvinacea adults tend to oviposit near the existing webs (Figure 4). 

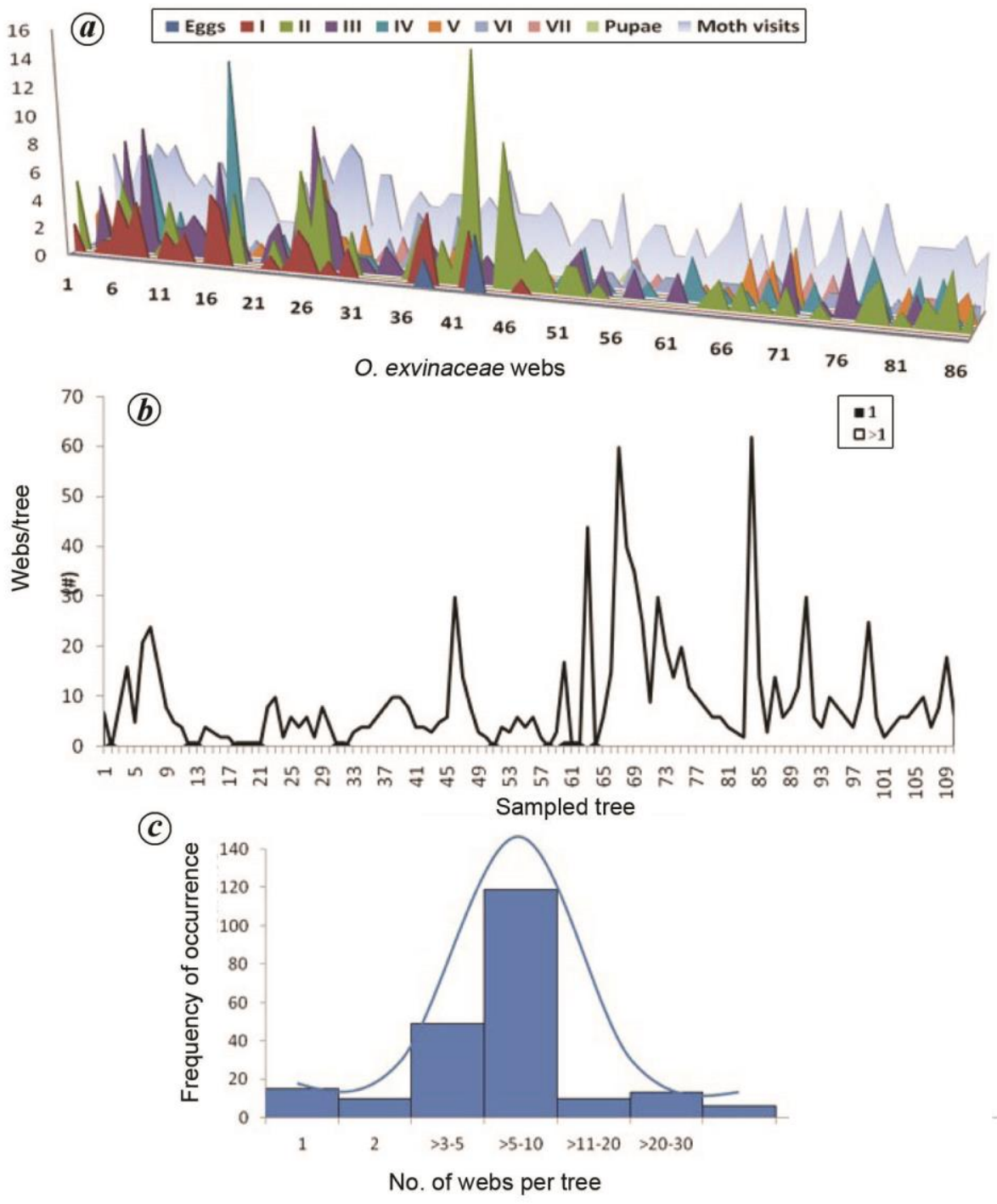

Figure 4. $\boldsymbol{a}$, Phenological composition of $O$. exvinacea webs and their relationship with expected moth visits. $\boldsymbol{b}$, Distribution pattern of web aggregation in a tree showing the occurrence of single (1) and multiple webs $(>1)$. $c$, Frequency distribution of multiple web occurrences/tree.

\section{Olfactometer assays}

Moths showed significant attraction to volatiles from middle-aged webs $(P=0.001)$ compared to those from young $(P=0.06)$, old $(P=0.36)$ and very old $(P=0.42)$ webs.

\section{Electrophysiology}

Mean EAG response exhibited by $O$. exvinacea gravid female antennae to volatiles of different categories of webs revealed that the EAG response (amplitude) was significantly higher $(0.78 \pm 0.05 \mathrm{mV}$; mean amplitude $\pm \mathrm{SE}$; $F=42.25 ; P<0.0001)$ for middle-aged web volatiles followed by newly formed web volatiles $(0.55 \pm 0.04 \mathrm{mV}$; mean amplitude \pm SE), compared to the others. The least
EAG response was noticed towards volatiles of very old webs $(0.18 \pm 0.05 \mathrm{mV}$; mean amplitude $\pm \mathrm{SE})$ followed by old webs $(0.03 \pm 0.01 \mathrm{mV}$; mean amplitude $\pm \mathrm{SE})$. GC-EAD revealed 13 electrophysiologically active cues that elicited antennal response in female $O$. exvinacea in the range $0.01-0.045 \mathrm{mV}$ (Table 1).

\section{Discussion}

Aggregation is a common phenomenon among vertebrate and invertebrate species/organisms in which social stimuli from conspecifics can alter the physiology, morphology or behaviour of individuals $\mathrm{s}^{12}$. The present study clearly proves that $O$. exvinacea tags along with conspecifics by ovipositing in or near the same web. The phenological asynchrony observed in each of the webs of $O$. exvinacea 
Table 1. GC-MS analysis of middle-aged web volatile compounds that elicited antennal response in gravid female of Orthaga exvinacea

\begin{tabular}{rlrc}
\hline RT & Compound identified & $\begin{array}{r}\text { Relative abundance } \\
(\%)\end{array}$ & $\begin{array}{r}\text { GC EAD response } \\
(\mathrm{mV})\end{array}$ \\
\hline 3.2 & $(E)$-2-hexenal & 2.941 & 0.020 \\
3.4 & cis-3-Hexen-1-ol & 2.941 & 0.020 \\
5.1 & $(Z$-3-hexen-1-ol, formate & 8.823 & 0.025 \\
5.7 & $\beta$-Ocimene & 14.705 & 0.025 \\
6.7 & $\beta$-Linalool & 2.941 & 0.020 \\
7.4 & Alloocimene & 3.529 & 0.022 \\
8.6 & cis-3-Hexenyl iso-butyrate & 2.352 & 0.020 \\
9.0 & $\delta$-Octalactone & 5.882 & 0.045 \\
11.2 & Methyleugenol & 17.647 & 0.010 \\
11.3 & Trans caryophyllene & 2.941 & 0.019 \\
11.8 & $(-)$ - $\alpha$-Gurjunene & 11.764 & 0.019 \\
12.4 & $\alpha$-Humulene & 17.647 & 0.020 \\
12.7 & Ledene & 5.882 & 0.010 \\
\hline
\end{tabular}

RT, Retention time; GC EAD, Gas chromatography electroantennodetection.

along with web aggregation within a tree explains that this moth has a tendency of social facilitation or conspecific attraction, which leads to variable cohort composition within the web and co-occurrence of multiple webs within the same tree. Considering the life-history traits of the webber moth, the presence of eggs, multiple larval stages and pupae that differ by several days within the same web supports the sequential oviposition behaviour of multiple number of gravid moths, rather than oviposition by a single moth. This was further supported by Taylor's fluctuations which showed the characteristic $b$ varying among different web groups. This explains the heterogeneity of web composition depending on age of the web. The largest value of $b$ (6.06) which was observed in younger webs and the progressive reduction in $b$ for other web groups implies that the web composition becomes comparatively more homogenous with age of the web. Among the different webs, inter-individual variability was tested by comparing the diversity and dispersion indices, which further supported the evident social facilitation in $O$. exvinacea. Olfactometer assays and electrophysiological studies also revealed significant response of female moths to volatiles of middle-aged webs. Moths comparatively showed the lowest response to volatiles of old and very old webs. Such conspecific attraction has been found to play a major role in the decision of habitat selection in several species, viz. lizards ${ }^{2}$, crabs $^{13}$ and birds ${ }^{14}$.

Several volatile cues that elicited antennal response in female $O$. exvinacea (Table 1) have been reported to have behavioural functions in other insects ${ }^{15}$. The chemical cues, namely (E)-hexenol, (Z)-3-hexen-1-ol, cis-3-

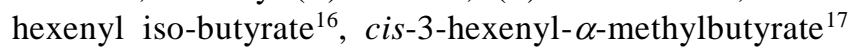
that elicited antennal response in $O$. exvinacea are known to belong to green leaf alcohols (GLVs) group. They are commonly emitted by wounded or stressed plants and play an important role in transferring information among insect-plant interactions ${ }^{18}$. Of several cues that elicited antennal response in $O$. exvinacea, $(E)$-hexenol is reported to act as an aggregation pheromone in Cimex lectularis and Euryderma rugosa at lower concentrations. Similarly, (Z)-3-hexen-1-ol which elicited antennal response in $O$. exvinacea, usually present in volatile emissions of wounded plants has been reported to prime the defences of neighbouring plants ${ }^{18}$. Other cues such as $\beta$-ocimene ${ }^{19}, \quad \beta$-linalool, trans-caryophyllene ${ }^{15}$, alloocimene $^{20}$, methyleugenol ${ }^{21}$, ledene ${ }^{22}$ and $\delta$-octalactone ${ }^{23}$, which elicited electrophysiological response in $O$. exvinacea are reported to serve as infochemicals in several insect-plant interactions.

The present study demonstrates the social facilitation habit of $O$. exvinacea moths during oviposition as they lay eggs near or in conspecific webs. This behaviour establishes the fact that presence of conspecifics can be an important habitat selection cue for $O$. exvinacea. In case of phytophagous insects, which are holometabolus, the decision of gravid female is critical for sustaining the fitness of its progeny ${ }^{24}$. Usually, the ovipositing female depends on several cues such as presence of secondary compounds, visual signals, natural enemies, mutualists, conspecific immatures and microclimate ${ }^{25}$ to choose a suitable oviposition site for the survival of its progeny. In spite of many factors that influence a female's choice, it is worth noting that the presence of conspecific immatures plays the most important role in the oviposition decision making process of $O$. exvinacea. Similar reproduction-linked social facilitation was noticed in German cockroach Blatella germanica, where social isolation slowed oocyte development, sexual maturation and sexual receptivity, whereas the presence of conspecifics accelerated reproduction ${ }^{12}$. The advantages of aggregation are well known for many species of lepidoptera, including protection against desiccation and predation ${ }^{26}$. Further, the gregarious larvae also benefit from aggregation 


\section{RESEARCH ARTICLES}

due to increased development and survival rates as well as reduced predation and parasitism ${ }^{27}$. Some studies also reported that aggregation may increase feeding efficiency by allowing early instars to overcome leaf toughness by a group attack ${ }^{28}$, by overwhelming the plant's defences ${ }^{29}$, or by laying down an architecture of silk that aids larval webbing ${ }^{30}$. Detailed explanation for this gregarious living pattern of $O$. exvinacea requires further studies to reveal the underlying possible implications of multiple cohorts (within a web) and webbings in the same tree. Further studies are being envisaged to identify the field attraction of potent chemical cues that aid the gravid females to oviposit in or near conspecific webs. Such chemical cues may aid in the future management programmes of O. exvinacea.

1. Wilson, E. O., Sociobiology, Harvard Belknap Press, Cambridge, Massachusetts and London, England, 1980, p. 384.

2. Stamps, J. A., Conspecific attraction and colonization in territorial species. Am. Nat., 1988, 131, 329-347.

3. Kurta, A., Social facilitation of foraging behaviour by the Hermit crab, Coenobita compressus, in Costa Rica. Biotropica, 1982, 14, 410.

4. Byers, D. L., Effect of cross proximity on progeny fitness in a rare and a common species of Eupatorium (Asteraceae). Am. J. Bot., 1998, 85, 644-653.

5. Mangel, M. and Roitberg, B. D., Dynamic information and host acceptance by a tephritid fruit fly. Ecol. Entomol., 1989, 14, 181189.

6. Katvala, M. and Kaitala, A., Conspecifics enhance egg production in an egg-carrying bug. Behav. Ecol., 2003, 14, 897-901.

7. Sisodiya, D. B., Patel, M. G. and Jhala, R. C., Biology of mango leaf webber Orthaga euadrusalis Walker (Lepidoptera: Pyralidae) on mango. In Proceedings of a National Seminar on Mango, Gujarat Agricultural University, Junagadh, 2003.

8. Verghese, A. et al., Evidence of a random ovipositional strategy by female fruit fly Bactrocera dorsalis (Tephritidae: Diptera) with reference to host quantum. Curr. Sci., 2011, 100, 246-249.

9. Kamala Jayathi, P. D. et al., Isolation and identification of host cues from mango, Mangifera indica that attract gravid female Oriental fruit fly, Bactrocera dorsalis, J. Chem. Ecol., 2012, 38, 361369 .

10. Piñero, J. C., Galizia, C. G. and Dorn, S., Synergistic behavioural responses of female oriental fruit moths (Lepidoptera: Tortricidae) to synthetic host-plant derived mixtures are mirrored by odourevoked calcium activity in their antennal lobes. J. Insect Physiol., 2008, 54, 333-343.

11. Baktavatsalam, N. et al., Autodetection in Helicoverpa armigera (Hubner). Curr. Sci., 2016, 110, 2261-2267.

12. Uzsak, A. and Schal, C., Sensory cues involved in social facilitation of reproduction in Blattella germanica females. PLOS ONE, 2013, 8; doi:10.1371/journal.pone.0055678.

13. Donahue, M. J., Conspecific cueing and growth-mortality tradeoffs jointly lead to conspecific attraction. Oecologia, 2006, 149, 33-43.

14. Doligez, B., Danchin, E. and Clobert, J., Public information and breeding habitat selection in a wild bird population. Science, 2002, 297, 1168-1170.
15. Kamala Jayanthi, P. D. et al., Functional diversity of infochemicals in agri-ecological networks. In Innovative Pest Management Approaches for the 21st Century: Harnessing Automated Unmanned Technologies (ed. Chakravarthy, A. K.), Springer Nature, Singapore, 2020, pp. 187-208.

16. Guo, H. and Wang, C., The ethological significance and olfactory detection of herbivore-induced plant volatiles in interactions of plants, herbivorous insects, and parasitoids. Arthropod-Plant Int., 2019, 13, 161-179.

17. Kessler, D. and Badwin, T., Making sense of nectar scents: the effects of nectar secondary metabolites on floral visitors of Nicotiana attenuate. Plant J., 2006, 49, 840-854.

18. Rika, O. et al., Intermittent exposure to traces of green leaf volatiles triggers the production of (Z)-3-hexen-1-yl acetate and (Z)-3hexen-1-ol in exposed plants. Plant Signal Behav., 2013, 8, 11.

19. Kang, Z. W. et al., Volatile $\beta$-ocimene can regulate developmental performance of peach aphid Myzus persicae through activation of defense responses in Chinese cabbage Brassica pekinensis. Front Plant Sci., 2018, 9, 708.

20. Kyutaro, K. et al., Volatile C6-aldehydes and allo-ocimene activate defense genes and induce resistance against Botrytis cinerea in Arabidopsis thaliana. Plant Cell Physiol., 2005, 46, 1093-1102.

21. Tan, K. H. and Nishida, R., Methyl eugenol: its occurrence, distribution, and role in nature, especially in relation to insect behaviour and pollination. J. Insect Sci., 2012, 12, 56.

22. Himanen, S. J., Blande, J. D. and Holopainen, J. K., Plant-emitted semi-volatiles shape the infochemical environment and herbivore resistance of heterospecific neighbours. Plant Signal. Behav., 2010, 5, 1234-1236.

23. Martin, T. et al., Repellent properties of $\delta$-octalactone against the tsetse fly, Glossina morsitans morsitans. J. Insect Sci., 2008, 8, 43.

24. Doak, P., Kareiva, P. and Kingsolver, J., Fitness consequences of choosy oviposition for a time-limited butterfly. Ecology, 2006, 87, 395-408.

25. Williams, K. S. and Gilbert, L. E., Insects as selective agents on plant vegetative morphology: egg mimicry reduces egg laying by butterflies. Science, 1981, 212, 467-469.

26. Francini, R. B. and Fraeitas, A. V. L., Aggregated oviposition in Actinote pellenea pellenea Hübner (Lepidoptera: Nymphalidae). J. Res. Lepid., 2010, 42, 74-78.

27. Denno, R. F. and Benre, B. Y., Aggregation facilitates larval growth in the neotropical nymphalid butterfly Chlosyne janais. Ecol. Entomol., 1997, 22, 133-141.

28. Ghent, A. W. A., Study of group-feeding behaviour of larvae of the jack-pine sawfly Neodiprion pratti banksianae Roh. Behaviour, 1960, 16, 110-148.

29. Storer, A. J., Ainhouse, D. W. and Speight, M. R., The effect of larval aggregation behaviour on larval growth of the spruce bark beetle Dendroctonus micans. Ecol. Entomol., 1997, 22, 109-115.

30. Rathcke, B. J. and Poole, R. W., Coevolutionary race continues: butterfly larval adaptation to plant trichomes. Science, 1975, 187, $175-176$.

ACKNOWLEDGEMENTS. We thank Indian Council of Agricultural Research, New Delhi for financial support through a National Fellow project, and the Director, Indian Institute of Horticultural Research, Bengaluru for providing the necessary facilities to carry out this work.

Received 7 April 2020; revised accepted 9 June 2020

doi: $10.18520 / \mathrm{cs} / \mathrm{v} 119 / \mathrm{i} 5 / 823-830$ 